\title{
Restoration of axial symmetry and its possible relation with restoration of chiral symmetry and deconfinement at finite temperature *
}

\author{
M. C. Ruivo, P. Costa, C. A. De Sousa \\ Centro de Física Computacional, Departamento de Física, Universidade de \\ Coimbra, P-3004 - 516 Coimbra, Portugal
}

(Received April 11, 2018)

The phase transitions characterized by deconfinement and restoration of chiral symmetry as well as the restoration of axial symmetry, at finite temperature, are investigated in the framework of SU(2) Polyakov-NambuJona-Lasinio (PNJL) models with the $\mathrm{U}_{A}(1)$ anomaly. The thermodynamics of the phase transitions, the topological susceptibility, the meson spectrum, and, in particular, the convergence of axial and chiral partners are analyzed, in the framework of the ordinary PNJL model and in its extension, the entangled Polyakov-Nambu-Jona-Lasinio (EPNJL) model. The latter incorporates entanglement between restoration of chiral symmetry and deconfinement.

PACS numbers: 11.30.Rd, 11.10.Wx, 11.55.Fv

An important field of research nowadays, both in experimental and theoretical physics, is the study of matter under extreme conditions of temperatures and/or densities. Quantum chromodynamics (QCD) predicts that, in this limit, matter becomes a plasma of deconfined quarks and gluons. In spite of the success of many aspects of the strong interaction physics, there are important features to clarify, and, in this context, the use of QCD inspired effective models has proven to be a useful approach.

The Polyakov-Nambu-Jona-Lasinio model is an effective model which respects important symmetries of the QCD action. It contains quarks as fundamental degrees of freedom allowing for a self-consistent description of chiral symmetry breaking and restoration. In addition, the coupling to the Polyakov loop allows to describe the (statistical) confinement/deconfinement phase transition by taking into account a static gluonic field in which quarks propagate [1, 2]. An important query on QCD thermodynamics is the

\footnotetext{
* Presented at eQCD2013
} 
proximity or coincidence of the chiral and deconfinement phase transitions. In this concern, lattice QCD results have been a matter of debate: the two phase transitions are reported to occur at distinct temperatures for $N_{f}=2+1$ flavors, but at the same temperature, $T_{c}=174(3)(6) \mathrm{MeV}$, for $N_{f}=2$ flavors [3]. This effect could be the result of strong correlations (entanglement) between the quark condensate and the Polyakov field, $\Phi$, an effect that is incorporated in the entangled Polyakov-Nambu-Jona-Lasinio model [4].

The restoration of the $\mathrm{U}_{A}(1)$ symmetry is also a longstanding question and phenomenological consequences for the nature of the phase transition are expected to occur, depending on the degree of anomaly present at the critical temperature. Moreover, the topological susceptibility, the meson axial chiral partners and the $\eta^{\prime}$ mass can exhibit signs of the restoration of the $\mathrm{U}_{A}(1)$ symmetry. Lattice calculations with three flavours have found evidence for the decrease of the topological susceptibility with temperature and for convergence of the meson correlators of chiral and axial partners when both symmetries are restored. The return of the 9th "prodigal" Goldstone boson has been a matter of debate and recent experimental results are compatible with a decrease of about $200 \mathrm{MeV}$ for the in medium mass of the $\eta^{\prime}$ meson (see [5] and references therein).

QCD inspired models have been used to study the restoration of chiral and axial symmetries, in particular the NJL model and its extended version, the PNJL model 6. In previous investigations, both in NJL model and PNJL SU(3) models [7, it has been found that observables related with the $\mathrm{U}_{A}(1)$ symmetry breaking vanish as a natural consequence of the effective restoration of chiral symmetry, provided a specific regularization is used [7]. However, the situation is different in the framework of the $\mathrm{SU}(2)$ models (see [5, 8]) where an additional mechanism of instantons suppression is needed.

The SU(2) PNJL Lagrangian with a 't Hooft interaction simulating the $\mathrm{U}_{A}(1)$ anomaly [2, 8] is given by :

$$
\mathcal{L}_{\mathcal{P N} \mathcal{J} \mathcal{L}}=\bar{q}\left(i \gamma^{\mu} D_{\mu}-\hat{m}\right) q+\mathcal{L}_{1}+\mathcal{L}_{2}-\mathcal{U}(\Phi[A], \bar{\Phi}[A] ; T),
$$

with two different interacting parts

$$
\begin{aligned}
& \mathcal{L}_{1}=g_{1}\left[(\bar{q} q)^{2}+\left(\bar{q} i \gamma_{5} \vec{\tau} q\right)^{2}+(\bar{q} \vec{\tau} q)^{2}+\left(\bar{q} i \gamma_{5} q\right)^{2}\right], \\
& \mathcal{L}_{2}=g_{2}\left[(\bar{q} q)^{2}+\left(\bar{q} i \gamma_{5} \vec{\tau} q\right)^{2}-(\bar{q} \vec{\tau} q)^{2}-\left(\bar{q} i \gamma_{5} q\right)^{2}\right],
\end{aligned}
$$

where $q=(u, d)$ are the Dirac and color fields, with two flavors, $N_{f}=2$, and three colors, $N_{c}=3$, the coupling coefficients $g_{1}$ and $g_{2}$ have dimension 
energy $^{-2}$, and $\hat{m}=\operatorname{diag}\left(m_{u}, m_{d}\right)$ is the current quark mass matrix, here being assumed that $m_{u}=m_{d}=m$. Both terms $\mathcal{L}_{1}$ and $\mathcal{L}_{2}$ are invariant upon $\mathrm{SU}(2)_{L} \otimes \mathrm{SU}(2)_{R} \otimes \mathrm{U}(1)$ type transformations, but $\mathcal{L}_{2}$, that can be written as a determinant (see [8]), is not invariant upon $\mathrm{U}_{A}(1)$ transformations.

The Lagrangian density (1) can be rewritten as

$$
\begin{aligned}
\mathcal{L}_{P N J L} & =\bar{q}\left(i \gamma^{\mu} D_{\mu}-\hat{m}\right) q \\
& +G_{s}\left[(\bar{q} q)^{2}+\left(\bar{q} i \gamma_{5} \vec{\tau} q\right)^{2}\right]+G_{a}\left[(\bar{q} \vec{\tau} q)^{2}+\left(\bar{q} i \gamma_{5} q\right)^{2}\right] \\
& -\mathcal{U}(\Phi[A], \bar{\Phi}[A] ; T)
\end{aligned}
$$

where $G_{s}=g_{1}+g_{2}$ and $G_{a}=g_{1}-g_{2}$ enter, respectively, in the propagators of the meson chiral partners $(\pi, \sigma)$ and $\left(\eta, a_{0}\right)$. We can redefine the coupling constants such as the set $\left(g_{1}, g_{2}\right)$ or $\left(G_{s}, G_{a}\right)$ will be replaced by $(G, c)$ in the following parametrization:

$$
G_{s}=g_{1}+g_{2}=G, \quad G_{a}=g_{1}-g_{2}=G(1-2 c),
$$

where $c \in\{0,1\}$ is a parameter that now specifies the degree of $\mathrm{U}_{A}(1)$ symmetry breaking. Notice that $g_{1}=G(1-c)$ is associated with chiral symmetry effects, while $g_{2}=c G$ is the anomaly coefficient and is here $25 \%$ of $g_{1}$. The parameters in the NJL sector of the model are $m=6 \mathrm{Mev}$, $\Lambda=590 \mathrm{Mev}, G \Lambda^{2}=2.435, c=0.2$, and were fixed by fitting lattice or experimental values for the pion mass, its decay constant, the quark condensate and the topological susceptibility, $\chi$. The masses of the $\sigma, \eta$ and $a_{0}$ mesons come as outputs (for detail see [5, 8, and references therein).

The quarks are coupled to the gauge sector via the covariant derivative $D^{\mu}=\partial^{\mu}-i A^{\mu}$, where $A^{\mu}(x)=g_{\text {Strong }} \mathcal{A}_{a}^{\mu}(x) \frac{\lambda_{a}}{2}$ and $\mathcal{A}_{a}^{\mu}$ is the $\mathrm{SU}_{c}(3)$ gauge field and $\lambda_{a}$ are the Gell-Mann matrices. The Polyakov loop $\Phi$ is the trace of the Polyakov line defined by: $\Phi=\frac{1}{N_{c}}\left\langle\left\langle\mathcal{P} \exp i \int_{0}^{\beta} d \tau A_{4}(\vec{x}, \tau)\right\rangle\right\rangle_{\beta}$.

The effective potential for the Polyakov loop is:

$$
\frac{\mathcal{U}(\Phi, \bar{\Phi} ; T)}{T^{4}}=-\frac{a(T)}{2} \bar{\Phi} \Phi+b(T) \ln \left[1-6 \bar{\Phi} \Phi+4\left(\bar{\Phi}^{3}+\Phi^{3}\right)-3(\bar{\Phi} \Phi)^{2}\right] .
$$

The parameters of the effective potential $\mathcal{U}$ have been fixed in order to reproduce the lattice data for the expectation value of the Polyakov loop and QCD thermodynamics in the pure gauge sector and are: $a_{0}=3.51, a_{1}=$ $-2.47, a_{2}=15.2, b_{3}=-1.75$; the parameter $T_{0}$ is the critical temperature for the deconfinement phase transition within a pure gauge approach and it was fixed to $270 \mathrm{MeV}$.

The PNJL model with the original $T_{0}=270 \mathrm{MeV}$ yields to a small difference between chiral and deconfinement transition temperatures. This value is however significant when we rescale $T_{0}$ to $210 \mathrm{MeV}$, derived by 


\begin{tabular}{||c||c|c|c|c|c||}
\hline \hline Scenario B & $\begin{array}{c}T_{0} \\
{[\mathrm{MeV}]}\end{array}$ & $\begin{array}{c}T_{\chi} \\
{[\mathrm{MeV}]}\end{array}$ & $\begin{array}{c}T_{d} \\
{[\mathrm{MeV}]}\end{array}$ & $\begin{array}{c}\Delta \\
-\end{array}$ & $\begin{array}{c}T_{\text {eff }} \\
{[\mathrm{MeV}]}\end{array}$ \\
\hline \hline PNJL & 210 & 215 & 177 & $18 \%$ & $\sim 250$ \\
& 270 & 237 & 219 & $8 \%$ & $\sim 300$ \\
\hline EPNJL & 170 & 173 & 173 & - & $\sim 200$ \\
& 270 & 223 & 223 & - & $\sim 300$ \\
\hline \hline
\end{tabular}

Table 1. Characteristic temperatures in PNJL and EPNJL model for different values of $T_{0}\left(\Delta=\left(T_{\chi}-T_{d}\right) / T_{\chi}\right)$. $T_{\text {eff }}$ is the temperature at which the effective restoration of both symmetries is achieved.

RG considerations so as to reproduce the lattice QCD result, $T_{d}=177$ $\mathrm{MeV}$. Consequently, the PNJL result is not consistent with lattice QCD data for the transition temperatures and entanglement between chiral and deconfinement transitions is weak in this model. The EPNJL model [4] incorporates entanglement by endowing the four-quark coupling vertex $G$ with a dependence on the Polyakov field, $\Phi$, in the form:

$$
G(\Phi)=G\left[1-\alpha_{1} \Phi \bar{\Phi}-\alpha_{2}\left(\Phi^{3}+\bar{\Phi}^{3}\right)\right]
$$

which respects chiral, $P, C$ and the extended center symmetries. The parameters $\alpha_{1}=\alpha_{2}=0.2$ and $T_{0}=170 \mathrm{MeV}$, taken from reference [4], were fixed to reproduce the available lattice QCD data.

As it can be seen in Table 1, in the PNJL model $T_{\chi}$ and $T_{d}$ never coincide but are closer for higher values of $T_{0}$, so it is adequate to choose $T_{0} \simeq 270$ $\mathrm{MeV}$. In the EPNJL model, where $\Delta=\left(T_{\chi}-T_{d}\right) / T_{\chi}=0$ by construction, we have more freedom to fix $T_{0}$ and a lower value is convenient $\left(T_{0} \simeq 170 \mathrm{MeV}\right)$ since it allows to reproduce lattice results for the critical temperature. The results are presented for scenario $\mathrm{B}$, for reasons that will be explained latter.

Notice that at finite $T$, due to the presence of the partition functions, the finite cutoff, $\Lambda$, used in the vacuum to regularize integrals, is not necessary and we make $\Lambda \longrightarrow \infty$. This procedure ensures that the pressure goes to the Stefan-Boltzmann limit and leads to a better description of several thermodynamic quantities. It has the disadvantage of leading to a sharp decrease of the quark condensates that do not vanish assimptoticaly and change sign unless a mechanism is imposed by hand to avoid this (for details see [7]). In SU(3) NJL and PNJL models the effective restoration of axial symmetry occurs as consequence of the full restoration of chiral symmetry. As a matter of fact, since in those models the 't Hooft interaction is a sixquark interaction, that in the usual approach is reduced to a four-quark 

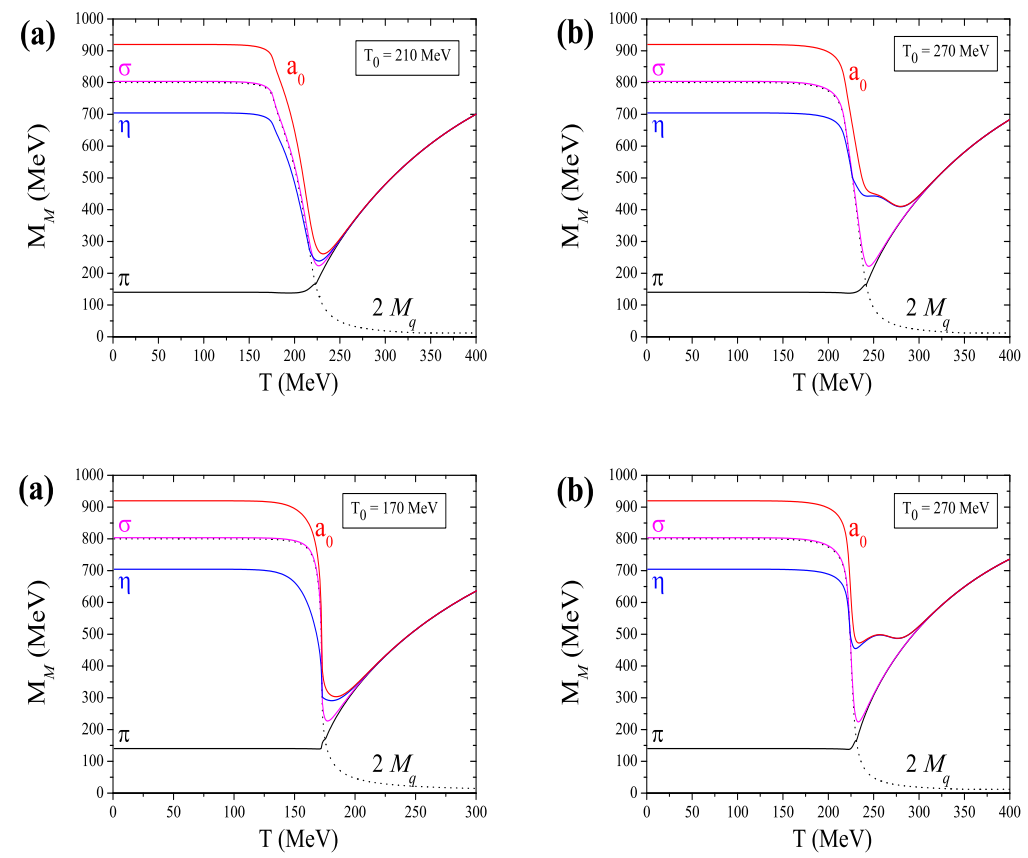

Fig. 1. Meson masses in PNJL (upper panels) and EPNJL (lower panels) models.

interaction by means of a Wick contraction of a quark-antiquark pair, we have a kind of "effective" 't Hooft coupling coefficient, $\tilde{G}_{D}=G_{D}<\bar{q} q>$, and, therefore, the anomaly effects disappear when the quark condensate anihilates. This is not so in the $\mathrm{SU}(2)$ models where the 't Hooft interaction is a four-quark vertex. In SU(2) models (even in the EPNJL model where the coupling vertices have a dependence of temperature through the Polyakov field, $\Phi)$ a fraction of the anomaly remains in the chiral restored phase because, although the topological susceptibility vanishes, the meson axial partners do not converge. In order to get this convergence it is enough that only $g_{2}$ acquires the adequate dependence on temperature and $g_{1}$ is kept constant (scenario A, see [5]).

Alternatively, we can use the redefinition of Eq. (15), with $c(T)=$ $0.2 f(T)$, where $f(T)=1 /\left(1+\exp \left(\left(T-T_{0}\right) / 10\right)\right)$. In the EPNJL model we have an equivalent explicit temperature dependence: $g_{1}(\Phi, T)=G(\Phi)(1-$ $c(T)$ ), $\quad g_{2}(\Phi, T)=G(\Phi) c(T)$ (scenario B). Both scenarios insure the vanishing of the anomaly and, therefore, the convergence of axial partners, but in scenario A, for both models, there is a unphysical region of negative pressures for low values of $T_{0}$. This problem that does not exist in scenario B, 
that allows more freedom in the choice of $T_{0}$. Here we will only discuss this last scenario.

In Fig. 1 we plot the PNJL results for the meson masses, considering two values of $T_{0}$. For $T_{0}=270 \mathrm{MeV}$, the convergence of chiral partners occurs first than that of axial partners, as usual, with $T_{\text {eff }} \approx 300 \mathrm{MeV}$. The new finding is that for $T_{0}=210 \mathrm{MeV}$ the chiral and axial partners get degenerate very closely and $T_{\text {eff }}$ is lower. Concerning the topological susceptibility, it allways vanishes, this effect being driven by the vanishing of the quark condensate.

The behavior of the meson masses and topological susceptibility in the EPNJL model is qualitatively similar to PNJL model, as it can be seen in Fig. 1. Here also the restoration of chiral and axial symmetries become closer for low values of $T_{0}$, but the temperatures for the effective restoration of symmetries are slightly lower. We conclude that in EPNJL there is entanglement between deconfinement and restoration of chiral symmetry but not with restoration of axial symmetry. Restoration of axial symmetry requires an adittional mechanism of instanton suppression.

Work supported by projects CERN/FP/116356/2010 and PTDC/FIS/ 100968/2008, developed under the initiative QREN financed by UE/FEDER through the Programme COMPETE - "Programa Operacional Factores de Competitividade".

\section{REFERENCES}

[1] P. N. Meisinger, and M. C. Ogilve, Phys. Lett. B379, 163 (1996); K. Fukushima, Phys. Lett. B591, 277 (2004); E. Megias, E. Ruiz Arriola, and L.L. Salcedo, Phys. Rev. D 74, 065005 (2006).

[2] C. Ratti, M. A. Thaler, and W. Weise, Phys. Rev. D 73, 014019 (2006); H. Hansen et al., Phys. Rev. D 75, 065004 (2007).

[3] A. Bazavov et al. Phys. Rev. D 85, 054503(2012); V. Bornyakov et al. Phys. Rev. D 82, 014504 (2010).

[4] Y. Sakai, T. Sasaki, H. Kouno, and M. Yahiro, Phys. Rev. D 82, 076003 (2010); J. Phys. G: Nucl. Part. Phys. 39, 035004 (2012).

[5] M. C. Ruivo, P. Costa, C. A. de Sousa, Phys. Rev. D 86, 116007 (2012).

[6] P. Costa, M. C. Ruivo, C. A. de Sousa, H. Hansen, and W. M. Alberico, Phys. Rev. D 79, 116003 (2009); P. Costa, M. C. Ruivo, C. A. de Sousa, and H. Hansen, Europhys. Lett. 86, 31001 (2009); P. Costa, M. C. Ruivo, C. A. de Sousa, and H. Hansen, Symmetry 2(3), 1338-1374 (2010).

[7] P. Costa, M. C. Ruivo and C. A. de Sousa, Phys. Rev. D 77, 096009 (2008); M. C. Ruivo et al., AIP Conf. Proc. 1257, 770 (2010).

[8] M. C. Ruivo, M. Santos, P. Costa, C. A. de Sousa, Phys. Rev. D 85, 036001 (2012). 